\title{
HILLE-WINTNER TYPE COMPARISON THEOREM FOR SELFADJOINT FOURTH ORDER LINEAR DIFFERENTIAL EQUATIONS
}

\author{
L. ERBE ${ }^{1}$
}

ABSTRaCr. The well-known Hille-Wintner Theorem for second order linear differential equations is extended to fourth order selfadjoint equations.

1. Introduction. Consider the selfadjoint fourth order equations

$$
\left(r(x) y^{\prime \prime}\right)^{\prime \prime}+p(x) y=0, \quad r(x)>0, p(x)>0,
$$

and

$$
\left(r(x) y^{\prime \prime}\right)^{\prime \prime}-p(x) y=0, \quad r(x)>0, p(x)>0 .
$$

The oscillation and nonoscillation properties of the solutions of equations (1.1) and (1.2) were the subject of an extensive and systematic study in the fundamental paper of Leighton and Nehari [9]. As noted there, the difference between the oscillatory behavior of (1.1) and (1.2) is very profound: Either all solutions of (1.1) are oscillatory or none are; however, (1.2) always has nonoscillatory solutions regardless of whether or not there are any oscillatory solutions.

An equation of the form (1.1) or (1.2) is said to be disconjugate on an interval $I$ in case no nontrivial solution has more than three zeros on $I$, counting multiplicities. In general, an $n$th order equation is disconjugate if no nontrivial solution has more than $n-1$ zeros, counting multiplicities. In this paper, we shall assume that the coefficients $r, p$ are continuous and positive on some half-line $I=[a,+\infty)$. A solution of (1.1) or (1.2) is said to be oscillatory if it has an infinite number of zeros in $[a,+\infty)$ and equation (1.1) or (1.2) is said to be oscillatory in case it has an oscillatory solution. If (1.1) or (1.2) is not oscillatory (i.e., if all solutions have only finitely many zeros), then the equation is disconjugate on some interval $\left[a_{1},+\infty\right)$, $a_{1}>a$ ([9]; see also [3]).

The oscillation properties of (1.1) and (1.2) are intimately connected with the conjugate and focal point theory as developed in [9], [3], [4], [5], [8]. In general, an $n$th order linear differential equation is said to be $(k, n-k)$ disconjugate on an interval $I$ in case no nontrivial solution has a zero of order $k$ followed by a zero of order $n-k$. In the case of equation $(1.1)$, disconjugacy is equivalent to $(3,1)$ disconjugacy (which, since $(1.1)$ is selfadjoint, is also equivalent to $(1,3)$ disconjugacy), and for equation (1.2), disconjugacy is equivalent to $(2,2)$ disconjugacy

Received by the editors September 25, 1979.

1980 Mathematics Subject Classification. Primary 34C10, 34C11.

${ }^{1}$ Research supported by NRC Grant A-07673. 
([9]). Similar restrictions apply to the index of disfocality. That is, (1.1) or (1.2) is said to be $(k, 4-k)$ disfocal on an interval $I$ for some $1<k<3$ in case there does not exist a solution $y$ with a zero of order $k$ followed by a zero of $\hat{y}^{(k)}$ of order $4-k$, where $\hat{y}^{(j)}=y^{(j)}$, for $j=0,1,2, \hat{y}^{(j)}=\left(r y^{\prime \prime}\right)^{(j-2)}, j=3,4$. Thus, in the case of $(1.1)$ the only type of focal points possible are $(3,1)$ and $(1,3)$ and for $(1.2)$ the only type possible is a $(2,2)$ focal point. (See [10], [5].)

2. Statement and proof of results. One of the most useful comparison theorems in the study of the oscillatory behavior of the second order linear equations

$$
\begin{gathered}
\left(r(x) y^{\prime}\right)^{\prime}+q(x) y=0, \\
\left(r_{1}(x) y^{\prime}\right)^{\prime}+q_{1}(x) y=0,
\end{gathered}
$$

aside from the Sturm Comparison Theorem, is the so called Hille-Wintner Comparison Theorem:

THEOREM 2.1 ([6], [13]). Let $r \equiv r_{1} \equiv 1, q, q_{1} \in C[a,+\infty)$, and let

$$
Q(x) \equiv \int_{x}^{\infty} q(t) d t, \quad Q_{1}(x) \equiv \int_{x}^{\infty} q_{1}(t) d t
$$

exists with $0 \leqslant Q_{1}(x) \leqslant Q(x)$ for all $x \in[a,+\infty)$. Then if $(2.1)$ is disconjugate on $[a,+\infty)$ so also is (2.2).

Theorem 2.1 was recently extended to the more general equations (2.1) and (2.2) by Butler [2] (who removed the boundedness assumption on $r(x)$ required in [12]) as follows:

THEOREM 2.2. Let $r, r_{1}, q, q_{1}$ be continuous on $[a,+\infty)$ such that

$$
Q(x)=\int_{x}^{\infty} q(t) d t, \quad Q_{1}(x)=\int_{x}^{\infty} q_{1}(t) d t
$$

exist, and such that $0<r(x) \leqslant r_{1}(x),\left|Q_{1}(x)\right|<Q(x)$ on $[a,+\infty)$. Then if $(2.1)$ is disconjugate on $[a,+\infty)$, so also is (2.2).

In contrast to the substantial number of comparison results which have been developed for (2.1) and (2.2) (cf. [14]) relatively few results are known for equations (1.1) and (1.2). It was shown in [9] that if $0<r(x) \leqslant r_{1}(x), 0<p_{1}(x)<p(x)$, and if (1.1) is disconjugate, then so also is

$$
\left(r_{1}(x) y^{\prime \prime}\right)^{\prime \prime}+p_{1}(x) y=0
$$

In fact, under the same assumptions on $r, r_{1}, p, p_{1}$, it was also shown that if (1.2) is disconjugate, then so also is

$$
\left(r_{1}(x) y^{\prime \prime}\right)^{\prime \prime}-p_{1}(x) y=0 .
$$

Various other comparison theorems were developed in [1], [7] and [9] and we refer the reader to [11] for a discussion of these and related topics. However, none of the results in the references cited deal with direct integral comparisons between equations of the form (1.1) and (2.3) or (1.2) and (2.4). The following two theorems provide these sorts of Hille-Wintner type comparisons. 
THEOREM 2.3. Let $r, r_{1}, p, p_{1} \in C[a,+\infty)$ satisfy

$$
0<r(x)<r_{1}(x), \quad p(x)>0, p_{1}(x)>0, x>a,
$$

and assume equation (1.1) is disconjugate on $[a,+\infty)$. Then if

$$
\int^{\infty} r_{1}^{-1} d t=+\infty, \quad P_{1}(x) \equiv \int_{x}^{\infty} p_{1}(t) d t<\int_{x}^{\infty} p(t) d t \equiv P(x), \quad x>a,
$$

equation (2.3) is disconjugate on $[a,+\infty)$.

Theorem 2.4. $r, r_{1}, p, p_{1} \in C[a,+\infty)$ satisfy (2.5) and assume (2.6) holds. Then if equation (1.2) is disconjugate on $[a,+\infty)$, then so also is equation (2.4).

Before beginning the proofs of the above theorems, we state the following two results whose proofs may be found in [4, Lemmas 3 and 6]:

Lemma 2.5. Assume $r, p>0$ and $\int^{\infty} r^{-1} d t=+\infty$. Then equation (1.1) (or (1.2)) is $(k, 4-k)$ disfocal iff it is $(k, 4-k)$ disconjugate.

LEMMA 2.6. Equation (1.1) (or (1.2)) is $(k, 4-k)$ disfocal in $(a, b)$ iff there exists a solution $y$ which satisfies

$$
\begin{gathered}
\hat{y}^{(j)}>0, \quad j=0,1, \ldots, k, \\
(-1)^{j-k} \hat{y}^{(j)}>0, \quad j=k+1, \ldots, 4,
\end{gathered}
$$

where $\hat{y}^{(j)}=y^{(j)}, j=0,1,2, \hat{y}^{(j)}=\left(r y^{\prime \prime}\right)^{(j-2)}, j=3,4$.

As a consequence of Lemmas 2.5, 2.6, and the remarks in the introduction, we note that if $\int^{\infty} r^{-1} d x=+\infty$, then equation (1.1) is disconjugate iff there exists a solution $y$ satisfying

$$
y>0, \quad y^{\prime}>0, \quad y^{\prime \prime}<0, \quad\left(r y^{\prime \prime}\right)^{\prime}>0, \quad\left(r y^{\prime \prime}\right)^{\prime \prime}<0,
$$

and equation (1.2) is disconjugate iff there exists a solution $y$ satisfying

$$
y>0, \quad y^{\prime}>0, \quad y^{\prime \prime}>0, \quad\left(r y^{\prime \prime}\right)^{\prime}<0, \quad\left(r y^{\prime \prime}\right)^{\prime \prime}>0 .
$$

Proof of Theorem 2.3. In view of the above remarks, let $y$ be a solution of (1.1) satisfying (2.7). We make the change of variable

$$
u_{1}=y^{\prime} / y, \quad u_{2}=r y^{\prime \prime} / y, \quad u_{3}=\left(r y^{\prime \prime}\right)^{\prime} / y
$$

so that

$$
\left\{\begin{array}{l}
u_{1}^{\prime}=r^{-1} u_{2}-u_{1}^{2}, \\
u_{2}^{\prime}=u_{3}-u_{1} u_{2}, \\
u_{3}^{\prime}=-p-u_{1} u_{3} .
\end{array}\right.
$$

It is easy to see that by (2.7), $u_{1} \rightarrow 0, u_{2} \rightarrow 0$, and $u_{3} \rightarrow 0$, as $x \rightarrow+\infty$ so that (2.10) can be replaced by the equivalent integral system

$$
\begin{aligned}
& u_{1}(x)=\int_{x}^{\infty}\left(u_{1}^{2}-r^{-1} u_{2}\right) d t \equiv \int_{x}^{\infty} g_{1}\left(u_{1}, u_{2}\right) d t \\
& u_{2}(x)=\int_{x}^{\infty}\left(u_{1} u_{2}-u_{3}\right) d t \equiv \int_{x}^{\infty} g_{2}\left(u_{1}, u_{2}, u_{3}\right) d t \\
& u_{3}(x)=\int_{x}^{\infty} u_{1} u_{3} d t+\int_{x}^{\infty} p d t \equiv \int_{x}^{\infty} g_{3}\left(u_{1}, u_{3}\right) d t+P(x)
\end{aligned}
$$


Since the solution $y$ satisfies (2.7), it follows that $u_{1}, u_{2}, u_{3}$ satisfy

$$
u_{1}>0, \quad u_{2}<0, \quad u_{3}>0 \text {. }
$$

Notice also, for $u_{1}, u_{2}, u_{3}$ in the range determined by (2.12), that $g_{1}\left(u_{1}, u_{2}\right)=u_{1}^{2}-$ $r^{-1} u_{2}$ is monotone increasing in $u_{1}$ and decreasing in $u_{2}, g_{2}\left(u_{1}, u_{2}, u_{3}\right)=u_{1} u_{2}-u_{3}$ is decreasing in $u_{1}$, increasing in $u_{2}$, and decreasing in $u_{3}$, and $g_{3}\left(u_{1}, u_{3}\right)=u_{1} u_{3}$ is increasing in both $u_{1}$ and $u_{3}$.

Consider now the system

$$
\begin{aligned}
& v_{1}(x)=\int_{x}^{\infty}\left(v_{1}^{2}-r_{1}^{-1} v_{2}\right) d t \equiv \int_{x}^{\infty} \hat{g}_{1}\left(v_{1}, v_{2}\right) d t \\
& v_{2}(x)=\int_{x}^{\infty}\left(v_{1} v_{2}-v_{3}\right) d t=\int_{x}^{\infty} g_{2}\left(v_{1}, v_{2}, v_{3}\right) d t \\
& v_{3}(x)=\int_{x}^{\infty} v_{1} v_{3} d t+\int_{x}^{0} p_{1} d t=\int_{x}^{\infty} g_{3}\left(v_{1}, v_{3}\right) d t+P_{1}(x)
\end{aligned}
$$

corresponding to equation (2.3). We show that (2.13) has a solution obtainable by successive approximations as follows: Define $v_{10}(x) \equiv u_{1}(x), v_{20}(x) \equiv u_{2}(x), v_{30}(x)$ $\equiv u_{3}(x)$ and for $n \geqslant 0$,

$$
\begin{aligned}
& v_{1 n+1}(x) \equiv \int_{x}^{\infty} \hat{g}_{1}\left(v_{1 n}(s), v_{2 n}(s)\right) d s \\
& v_{2 n+1}(x) \equiv \int_{x}^{\infty} g_{2}\left(v_{1 n}(s), v_{2 n}(s), v_{3 n}(s)\right) d s, \\
& v_{3 n+1}(x) \equiv \int_{x}^{\infty} g_{3}\left(v_{1 n}(s), v_{3 n}(s)\right) d s+P_{1}(x) .
\end{aligned}
$$

By induction we may show using the monotone properties of the integrands that

$$
0<v_{1 n+1}<v_{1 n}<u_{1}, \quad 0>v_{2 n+1}>v_{2 n}>u_{2}, \quad 0<v_{3 n+1}<v_{3 n}<u_{3}
$$

for all $n>0$. The fact that $\left|v_{i n}\right|>0, i=1,2,3$, follows from the fact that $p_{1}(x) \neq 0$ for all large $x$. Hence, $\left\{v_{1 n}\right\},\left\{v_{2 n}\right\},\left\{v_{3 n}\right\}$ converge monotonically and uniformly on compact subintervals of $[a,+\infty)$ to a solution $\hat{v}_{1}, \hat{v}_{2}, \hat{v}_{3}$ of system (2.13). It follows also that $\hat{v}_{1}(x)>0, \hat{v}_{2}(x)<0, \hat{v}_{3}(x)>0, x>a$ so defining $z(x) \equiv \exp \left(\int_{a}^{x} \hat{v}_{1}(s) d s\right)$, we see that $z>0, x>a$, and $z$ is a solution of (2.3) which satisfies $z>0, z^{\prime}>0, z^{\prime \prime}<0,\left(r_{1} z^{\prime \prime}\right)^{\prime}>0,\left(r_{1} z^{\prime \prime}\right)^{\prime \prime}<0$. Therefore, by Lemmas 2.5 and 2.6, we conclude that equation (2.3) is disconjugate. This completes the proof of Theorem 2.3.

ProOF OF THEOREM 2.4. Reflecting the change between the nature of the solutions of (1.1) and (1.2) is the method of proof for Theorem 2.4 which is entirely different from that for Theorem 2.3. We first need the following Lemma which gives a necessary and sufficient condition for disconjugacy of (1.2) based on two related second order equations.

LEMMA 2.7. Equation (1.2) is disconjugate on $[a,+\infty)$ iff there exists a positive function $\sigma \in C^{1}[a,+\infty)$ such that both of the equations

$$
\left(r u^{\prime}\right)^{\prime}+\sigma u=0, \quad\left(\sigma v^{\prime}\right)^{\prime}+p v=0
$$

are disconjugate in $[a,+\infty)$. 
Proof. If there exists $\sigma \in C^{\prime}[a,+\infty)$, $\sigma>0$, such that (2.15) is disconjugate, then (1.2) is disconjugate (this is just Theorem 6.3 of [9]). On the other hand, if equation (1.2) is disconjugate, then it is $(2,2)$ disconjugate and hence by Lemmas 2.5 and 2.6 there exists a solution $y$ which satisfies.

$$
y>0, \quad y^{\prime}>0, \quad y^{\prime \prime}>0, \quad\left(r y^{\prime \prime}\right)^{\prime}<0, \quad\left(r y^{\prime \prime}\right)^{\prime \prime}>0 .
$$

Letting $\sigma=-\left(r y^{\prime \prime}\right)^{\prime} / y^{\prime \prime}$ we see that $u=y^{\prime}$ and $v=y$ are positive solutions of (2.15) and hence both equations are disconjugate in $[a,+\infty)$. This proves the Lemma. Suppose now that $r, r_{1}, p, p_{1}$ satisfy (2.5) and assume (2.6) holds. If equation (1.2) is disconjugate, then by Lemma 2.7, there exists $\sigma>0, \sigma \in C^{\prime}[a,+\infty)$ such that $\left(r u^{\prime}\right)^{\prime}+\sigma u=0$ and $\left(\sigma v^{\prime}\right)^{\prime}+p v=0$ are both disconjugate. Now by the Sturm Comparison Theorem, since $0<r<r_{1}$, it follows that $\left(r_{1} u^{\prime}\right)^{\prime}+\sigma u=0$ is also disconjugate. Moreover, since $0 \leqslant \int_{x}^{\infty} p_{1} d t \leqslant \int_{x}^{\infty} p d t$, it follows by Theorem 2.2 that $\left(\sigma v^{\prime}\right)^{\prime}+p_{1} v=0$ is likewise disconjugate. Therefore, by Lemma 2.7 we conclude that (2.4) is disconjugate. This completes the proof of Theorem 2.4.

As applications and to demonstrate sharpness, we state the following two corollaries:

COROLlary 2.8. The equation

$$
y^{(4)}-p_{1}(x) y=0, \quad p>0,
$$

is disconjugate in $[a,+\infty)$ if

$$
x^{3} \int_{x}^{\infty} p_{1}(t) d t \leqslant \frac{3}{16}, \quad x>a .
$$

To prove this, we use the nonoscillatory Euler equation $y^{(4)}-9 x-4 / 16=0$.

Similarly, using the nonoscillatory Euler equation $y^{(4)}+x^{-4} y=0$, we establish

COROLlary 2.9. The equation

$$
y^{(4)}+p_{1}(x) y=0, \quad p_{1}>0
$$

is disconjugate in $[a,+\infty)$ if

$$
x^{3} \int_{x}^{\infty} p_{1}(t) d t<\frac{1}{3}, \quad x>a .
$$

\section{REFERENCES}

1. J. H. Barrett, Oscillation theory of ordinary differential equations, Advances in Math. 3 (1969), 415-509.

2. G. J. Butler, Hille-Wintner comparison theorems for second order ordinary differential equations, Proc. Amer. Math. Soc. 76 (1979), 51-59.

3. U. Elias, Nonoscillation and eventual disconjugacy, Proc. Amer. Math. Soc. 66 (1977), 269-275.

4. __ Oscillatory solutions and extremal points for a linear differential equation, Arch. Rational Mech. Anal. 71 (1979), 177-198.

5. Focal points for a linear differential equation whose coefficients are of constant signs, Trans. Amer. Math. Soc. 249 (1979), 187-202.

6. E. Hille, Nonoscillation theorems, Trans. Amer. Math. Soc. 64 (1948), 234-252.

7. H. C. Howard, Oscillation criteria for fourth order linear differential equations, Trans. Amer. Math. Soc. 96 (1960), 296-311.

8. K. Kreith, Comparison theorems for a class of selfadjoint fourth order differential equations, Proc. Amer. Math. Soc. 67 (1977), 67-73. 
9. W. Leighton and Z. Nehari, On the oscillation of solutions of selfadjoint linear differential equations of the fourth order, Trans. Amer. Math. Soc. 89 (1958), 325-377.

10. Z. Nehari, Green's functions and disconjugacy, Arch. Rational Mech. Anal. 62 (1976), 53-76.

11. C. A. Swanson, Comparison and ascillation theory of linear differential equations, Academic Press, New York, 1968.

12. C. T. Taam, Nonascillatory differential equations, Duke Math. J. 19 (1952), $493-497$.

13. A. Wintner, On the comparison theorem of Kneser-Hille, Math. Scand. 5 (1957), 255-260.

14. J. S. W. Wong, Oscillation and nonascillation of solutions of second order linear differential equations with integrable coefficients, Trans. Amer. Math. Soc. 144 (1969), 197-215.

Departmient of Mathematics, University of Alberta, Edmonton, Alberta, Canada 